\title{
Role of underappreciated vectors in malaria transmission in an endemic region of Bangladesh-India border
}

Hasan Mohammad Al-Amin ${ }^{1}$, Rubayet Elahi ${ }^{1,2}$, Abu Naser Mohon 1,3, Mohammad Abdullah Heel Kafi ${ }^{1}$, Sumit Chakma', Jennifer S Lord ${ }^{4}$, Wasif A Khan ${ }^{1}$, Rashidul Haque ${ }^{1}$, Douglas E Norris ${ }^{5}$ and Mohammad Shafiul Alam ${ }^{{ }^{*}}$

\begin{abstract}
Background: Despite the efforts of the National Malaria Control Programme, malaria remains as an important public health problem in Bangladesh, particularly in the south-eastern region bordering India. Successful malaria control strategies rely on a detailed understanding of the underlying causes of malaria transmission. Here, an entomological survey was conducted in a malaria endemic area of Bangladesh bordering India to investigate the Anopheles mosquito community and assess their Plasmodium infection status.

Methods: Monthly entomological collections were undertaken from October 2010 to September 2011 in five villages in the Matiranga sub-district, Khagrachari district in Bangladesh, bordering the Indian State of Tripura. CDC miniature light traps were placed inside houses to collect adult Anopheles mosquitoes. Following morphological and molecular identification of the female Anopheles mosquitoes collected, they were screened for circumsporozoite proteins (CSP) of Plasmodium falciparum (Pf), Plasmodium vivax-210 (Pv-210) and Plasmodium vivax-247 (Pv-247), by ELISA to determine natural infection rates. Variation in Anopheles species composition, relative abundance and Plasmodium infection rates were analysed between sampled villages.
\end{abstract}

Results: A total of 2,027 female Anopheles were collected, belonging to 20 species. Anopheles nivipes was the most abundant species in our test villages during the peak malaria transmission season, and was observed sympatrically with An. philippinensis in the studied area. However, in the dry off-peak season, An. jeyporiensis was the most abundant species. Shannon's diversity index was highest in October (2.12) and evenness was highest in May (0.91). The CSP ELISA positive rate overall was 0.44\%. Anopheles karwari $(n=2)$, An. barbirostris s.l. $(n=1)$ and An. vagus $(n=1)$ were recorded positive for Pf. Anopheles kochi $(n=1)$ was positive for Pv-210 while An. umbrosus $(n=1)$, An. nivipes $(n=1)$ and $A n$. kochi $(n=1)$ were positive for Pv-247. A mixed infection of Pf and Pv-247 was detected in An. barbirostris s.l..

Conclusion: High diversity of Anopheles species was observed in areas close to the international border where species that were underestimated for malaria transmission significantly outnumbered principal vector species and these may play a significantly heightened role in malaria transmission.

Keywords: Anopheles, Plasmodium, Malaria, Species diversity, Vector, Matiranga, Bangladesh

\footnotetext{
* Correspondence: shafiul@icddrb.org

${ }^{1}$ International Centre for Diarrhoeal Disease Research Bangladesh (icddr,b), 68

Shaheed Tajuddin Ahmed Sarani, Mohakhali, Dhaka 1212, Bangladesh

Full list of author information is available at the end of the article
} 


\section{Background}

There were an estimated 198 million cases and 584,000 deaths attributed to malaria globally during 2013 [1]. In Bangladesh, malaria represents a major public health concern with approximately 27,000 cases and 15 deaths reported during 2013 [2]. Malaria in Bangladesh is markedly seasonal, where the warm and wet months of MayOctober define the peak malaria transmission season, and the dry and cooler months of November-April define the off-peak season [3]. Although once widespread throughout the country, malaria is now restricted to 13 districts bordering India and Myanmar [4]. In particular, Khagrachari, Bandarban and Rangamati districts along with Cox's Bazar districts in the Chittagong Hill Tracts (CHT), account for $80 \%$ of malaria cases of the country [5].

Caused predominantly by Plasmodium falciparum (Pf), the epidemiology and therefore control of malaria in this region is complicated by high local Anopheles vector diversity and the fact that most vectors are members of species complexes. These species include Anopheles (Cellia) vagus Dönitz, An. (C.) jeyporiensis James and $A n$. (C.) nivipes Theobald in addition to known major vectors $A n$. (C.) baimaii Sallum \& Peyton, An. (C.) minimus complex Theobald, An. (C.) philippinensis Ludlow, An. (C.) epiroticus Linton \& Harbach, An. (C.) aconitus Dönitz and $A n$. (C.) annularis van der Wulp [6,7].

Topologically, the CHT districts vary from other regions of the country being typified by ranges of forested hills and associated valleys with rivers and small lakes [8] that provide abundant oviposition sites for potential vector species. With the presence of a diverse vector community on both sides of the Bangladesh-India border, vector control interventions in this region are currently reported as inadequate [7-9]. The remote and hilly terrain of the border belt areas makes malaria transmission here harder to interrupt. Moreover, unrestricted movement of people across the border for occupation and trade increase the complexity of transmission. Taken together, this has led to malaria persistence in the villages adjacent to the border [10].

Over the decades, the abundance of different Anopheles species throughout the endemic areas of Bangladesh has changed [6-8]. Deforestation and changing agricultural practices in the CHT [11] may explain the altered abundance of Anopheles species and their vectorial role [7], once prevalent species have been documented as rare in more recent studies $[7,8]$. However, only a few data on mosquito diversity and abundance in the hilly border belt areas are available [7,8,12]. Prediction of malaria epidemics and plans for control programmes are greatly dependent on knowledge of vector dynamics and distribution [13] because disease transmission depends on the presence or absence of vector species and their specific behaviours [14]. A better understanding of vector species is necessary for any successful integrated control strategy [15]. This entomological survey was undertaken in a malaria endemic area of CHT, near the BangladeshIndia border to reveal the diversity and abundance of Anopheles species over the study period, the variation of Anopheles mosquito diversity and abundance among villages and distances from the international border and their Plasmodium infection status.

\section{Methods}

\section{Study area}

The study was conducted in the Matiranga sub-district $\left(23^{\circ} 02^{\prime} 19^{\prime \prime} \mathrm{N}, 91^{\circ} 52^{\prime} 36^{\prime \prime} \mathrm{E}\right)$ of Khagrachari district, adjacent to the Bangladesh-India border (Figure 1). Matiranga, covers approximately $495 \mathrm{~km}^{2}$ and supports 126,477 people at a density of 255.3 per $\mathrm{km}^{2}$ [16]. The region has a tropical monsoon climate. During the study period, there was an average rainfall of $217.17 \mathrm{~mm}$ and annual average maximum and minimum temperatures of $30.1^{\circ} \mathrm{C}$ and $20.6^{\circ} \mathrm{C}$, respectively and an average humidity of $79.25 \%$ (data obtained from a weather station situated at Rangamati which is 15 kilometres away from the study site) were recorded. During surveys, the landscape comprised of paddy fields on the plains and unused shrub lands or teak plantations in hilly areas.

Entomological investigations were conducted in the following five villages: Azoddha, Belchhari, Aambagan, Khedachhara and Natunpara (listed by proximity to the Bangladesh-India international border). Bengali and indigenous people reside in mixed communities, in houses constructed mostly of mud and bamboo. Villages were selected based on high malaria endemicity reports, according to data obtained from the Upazilla Health Complex (UHC) of Matiranga.

\section{Collection of Anopheles mosquitoes}

Monthly entomological surveys were undertaken between October 2010 and September 2011 in each village. Adult mosquitoes were collected from the sleeping room of houses using battery operated CDC miniature light traps (Model: 1012, Origin: John W. Hock Inc, USA) between 1800 and 0600 hours. All houses in each village were numbered and an online randomizer (http://www. randomizer.org/form.htm) was used to randomly determine which house to sample.

Each month, five light traps were installed in a village for one night. This enabled five consecutive trap nights in five villages giving 25 traps per month. A total of 60 trap nights were run in each village. Thus, in five villages a total of 300 trap nights were run.

\section{Processing of mosquitoes}

Mosquitoes were taken to the International Centre for Diarrhoeal Disease Research Bangladesh (icddr,b), field 


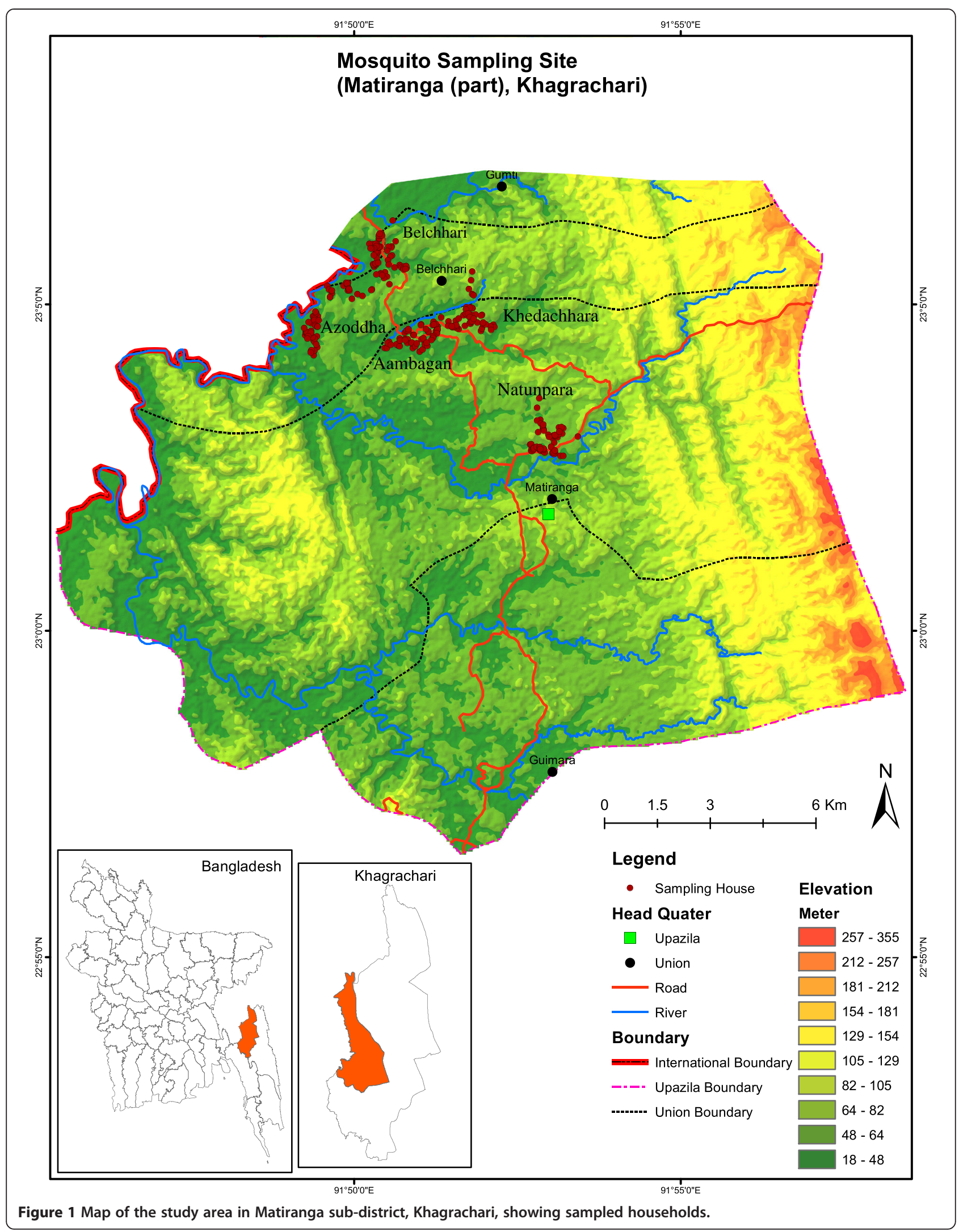


laboratory in Matiranga, killed by chloroform and subsequently identified. Mosquito identification to species level was undertaken using a stereomicroscope, according to Peyton et al. [17] and Nagpal and Sharma [18]. After identification, each mosquito was preserved in a cryo-vial (labelled with species name, trap number and date) containing desiccant until further analyses.

\section{Mosquito sample preparation}

After field identification, the mosquitoes were taken to the Parasitology Laboratory at icddr,b, Dhaka for further analyses. Each mosquito was dissected into head + thorax and abdomen and homogenized individually in $200 \mu \mathrm{L}$ and $100 \mu \mathrm{L}$ of phosphate buffered saline (PBS), respectively. The abdomen homogenate was used for PCR analysis to confirm species identification after DNA extraction as described below and from the head + thorax homogenate $150 \mu \mathrm{L}$ was used for circumsporozoite protein specific enzyme-linked immunosorbent assay (CSP ELISA).

\section{Molecular identification of mosquitoes}

DNA was extracted from mosquito homogenates using the CHELEX ${ }^{\oplus}$ protocol of Lardeux et al. [19] with the following modifications. Briefly, $50 \mu \mathrm{L}^{\text {CHELEX }}{ }^{\circ}-100$ $(50 \% \mathrm{v} / \mathrm{v})$ solution was added to $100 \mu \mathrm{L}$ mosquito homogenate. After an incubation of 15 minutes at $100^{\circ} \mathrm{C}$, the mixture was centrifuged at maximum speed for $5 \mathrm{mi}-$ nutes. $30 \mu \mathrm{L}$ of supernatant was transferred to a new tube and $2 \mu \mathrm{L}$ Proteinase- $\mathrm{K}$ solution was added. After subsequent incubations at $37^{\circ} \mathrm{C}$ for 45 minutes and at $100^{\circ} \mathrm{C}$ for 5 minutes the DNA was ready. PCR was undertaken with the extracted mosquito DNA to confirm the taxonomic identification where an established protocol was available. Protocols previously described by Huong et al. [20] for confirmation of An. baimaii, Phuc et al. [21], for species belonging to An. minimus complex and the Myzomyia Series, as well as two different protocols of Walton et al. [22,23] for confirmation of An. annularis and members of the An. (C.) maculatus Theobald group were used. A $51000^{\circ}$ Thermal Cycler (Bio-Rad Laboratories, Inc., Hercules, CA, USA) was used for amplification reactions. Post amplification PCR products were electrophoresed on ethidium bromidestained $1.5 \%$ agarose gels, along with Invitrogen 100 base pair (bp) molecular mass marker (Life Technologies, NY, USA) and visualized under UV illumination.

\section{CSP ELISA}

ELISAs were completed according to Wirtz et al. [24] with slight modification (phosphate buffered saline instead of blocking buffer was used to prepare mosquito homogenates) to detect CSP of Pf and two CSP polymorphs of $P$. vivax (Pv-210 and Pv-247). Field caught male mosquitoes were used as negative controls. The positive controls and monoclonal antibodies were obtained from the Centers for Disease Control and Prevention (CDC), Atlanta, USA. The Optical Density (OD) was measured at $410 \mathrm{~nm}$ in a Bio-Rad ELISA plate reader, 60 minutes after substrate addition. A cut-off value at least twice the mean $\mathrm{OD}$ of the negative controls was considered as positive. All positive samples were confirmed by repeated ELISA.

\section{Data analysis}

Mosquito species diversity and evenness in different months and villages were calculated by using the Shannon's diversity index $\left(\mathrm{H}^{\prime}\right)$ and Shannon's evenness index (E) [25]. Shannon's diversity index and evenness denote the faunal diversity of a community. Since the sample size of Plasmodium positive mosquitoes was too small to allow statistical analyses, mosquito light trap count data of the ten most common Anopheles species and whether Plasmodium positive was used to compare among villages. The overall variation of mosquito abundance was compared among villages using risk ratio by negative binomial regression because the mean number of mosquitoes is greater than the variance per village. According to Ejercito and Urbino [26] and Nagpal and Sharma [18] the flight range of the ten most common Anopheles species collected in this study is generally within one kilometre. Therefore, the distance was taken into consideration to further explore which village shows the maximum variation in terms of mosquito abundance. Villages were compared after adjusting the distance of villages from the international border. Similar analysis was undertaken for each mosquito species to see variation among villages. All data analysis was conducted in $\mathrm{R}$ (version 3.0.2) [27]. The geographic positions of the sampled houses were taken with a GPS receiver (Garmin 60CS). ArcGIS 10 (ESRI, CA, USA) was used for map preparation.

\section{Ethical approval}

Written consents were obtained from the households where mosquito collections were conducted. Ethical approval was obtained from Research Review Committee and Ethical Review Committee of icddr,b for this study.

\section{Results}

A total of 2,027 female Anopheles mosquitoes were caught (6.76 mosquitoes/trap night, standard error 1.46). Twenty species were confirmed based on taxonomic characteristics and molecular diagnosis for available species complexes. Of 292 mosquitoes morphologically identified as An. philippinensis, 247 were molecularly identified as An. nivipes, with the reminder (45) being An. philippinensis. In the present study, three of 19 
specimens morphologically identified as An. minimus were molecularly identified as $A n$. (C.) varuna Iyengar. The remaining 16 specimens were confirmed as An. minimus (An. minimus former species A) by PCR. Of $35 \mathrm{An}$. varuna specimens identified by morphology, one specimen was revised to An. minimus after molecular analysis. Concordance between morphological and molecular identification was observed for all other specimens and species.

Anopheles jeyporiensis was observed as the dominant species overall $(\mathrm{n}=505,24.91 \%)$, due primarily to a high trap count $(n=267)$ during November (Table 1 and Figure 2). The next most numerous species were $A n$. nivipes ( $\mathrm{n}=330), A n$. (C.) kochi Dönitz $(\mathrm{n}=259), A n$. (C.) karwari James $(\mathrm{n}=219)$ and An. vagus $(\mathrm{n}=217)$ (Table 1). A sharp decrease in the mean number of mosquitoes was observed from January (which is considered as the dry season) to May, the first month of the wet season in Bangladesh. Mean number of mosquitoes began to increase in June (Figure 2). Interestingly, the abundance of traditionally accepted primary vector species such as An. baimaii, An. philippinensis and An. minimus was observed to be much less than newly documented or secondary vector mosquitoes such as $A n$. jeyporiensis, $A n$. vagus and An. nivipes (Table 1 ).

Species richness was highest (17) in November and lowest (4) in February. However, Shannon's diversity index was highest in October $\left(\mathrm{H}^{\prime}=2.12\right)$ (Table 2). Anopheles nivipes $(\mathrm{n}=233)$ was recorded to be the dominant species during peak malaria transmission season, followed by $A n$. (A.) peditaeniatus Leicester $(\mathrm{n}=112)$, An. vagus $(\mathrm{n}=105)$, An. karwari $(\mathrm{n}=99)$, An. kochi $(\mathrm{n}=$ 95), and An. jeyporiensis $(\mathrm{n}=93)$ (Figure 3). However, during the off-peak season, An. jeyporiensis was the dominant species $(\mathrm{n}=412)$, followed by An. kochi $(\mathrm{n}=$ $164)$ and An. karwari $(\mathrm{n}=120)$.

CSP ELISA was carried out on all 2,027 females collected. CSP positivity was observed in nine mosquitoes $(0.44 \%)$ belonging to six species, which were collected from eight houses (Table 3). Plasmodium falciparum infection was reported from four mosquitoes $(0.19 \%)$ : one An. (A.) barbirostris s.l. van der Wulp, one An. vagus

Table 1 List of Anopheles species collected from five villages (300 trap nights) of Matiranga sub-district, Khagrachari from October 2010 to September 2011

\begin{tabular}{|c|c|c|c|c|c|c|c|}
\hline \multirow{2}{*}{$\begin{array}{l}\text { Anopheles } \\
\text { species }\end{array}$} & \multicolumn{5}{|c|}{ Number (\%) of Anopheles species in different villages } & \multirow[t]{2}{*}{ Number } & \multirow[t]{2}{*}{$(\%)$} \\
\hline & Natunpara & Aambagan & Khedachhara & Belchhari & Azoddha & & \\
\hline \multicolumn{8}{|l|}{ Subgenus Cellia } \\
\hline An. aconitus & $1(0.31)$ & $3(1.13)$ & $2(0.67)$ & 0 & $7(0.75)$ & 13 & 0.64 \\
\hline An. annularis & 0 & 0 & 0 & 0 & $1(0.11)$ & 1 & 0.05 \\
\hline An. baimaii & 0 & 0 & $3(1.00)$ & 0 & $1(0.11)$ & 4 & 0.20 \\
\hline An. jamesii & 0 & $1(0.38)$ & $3(1.00)$ & 0 & $5(0.53)$ & 9 & 0.44 \\
\hline An. jeyporiensis & $67(20.81)$ & $73(27.44)$ & $33(11.04)$ & 37 (18.05) & 295 (31.55) & 505 & 24.91 \\
\hline An. karwari* & 45 (13.97) & $37(13.91)$ & $48(16.05)$ & $24(11.71)$ & $65(6.95)$ & 219 & 10.80 \\
\hline An. kochi* & $40(12.42)$ & $20(7.52)$ & $18(6.02)$ & 37 (18.05) & $144(15.40)$ & 259 & 12.78 \\
\hline An. maculatus & 0 & $2(0.75)$ & $5(1.67)$ & $1(0.49)$ & $9(0.96)$ & 17 & 0.84 \\
\hline An. minimus & 0 & 0 & $3(1.00)$ & 0 & $14(1.50)$ & 17 & 0.84 \\
\hline An. nivipes* & $29(9.06)$ & $51(19.17)$ & $60(20.07)$ & $52(25.37)$ & 138 (14.76) & 330 & 16.18 \\
\hline An. philippinensis & $3(0.93)$ & $8(3.01)$ & $7(2.34)$ & $2(0.98)$ & $25(2.67)$ & 45 & 2.22 \\
\hline An. subpictus & $2(0.62)$ & 0 & 0 & 0 & $10(1.07)$ & 12 & 0.59 \\
\hline An. tessellatus & 0 & 0 & 0 & 0 & $1(0.11)$ & 1 & 0.05 \\
\hline An. vagus* & $53(16.46)$ & $26(9.77)$ & $11(3.68)$ & $24(11.71)$ & 103 (11.02) & 217 & 10.71 \\
\hline An. varuna & $4(1.24)$ & $3(1.13)$ & $2(0.67)$ & $1(0.49)$ & $27(2.89)$ & 37 & 1.83 \\
\hline An. willmori & $1(0.31)$ & 0 & 0 & 0 & $1(0.11)$ & 2 & 0.10 \\
\hline \multicolumn{8}{|l|}{ Subgenus Anopheles } \\
\hline An. barbirostris s.l.* & $16(4.97)$ & $11(4.14)$ & $13(4.35)$ & $8(3.90)$ & $45(4.81)$ & 93 & 4.59 \\
\hline An. nigerrimus & $19(5.90)$ & $4(1.50)$ & $24(8.03)$ & $5(2.44)$ & $8(0.86)$ & 60 & 2.97 \\
\hline An. peditaeniatus & $34(10.56)$ & $21(7.89)$ & $61(20.40)$ & $11(5.37)$ & $23(2.46)$ & 150 & 7.40 \\
\hline An. umbrosus* & $8(2.48)$ & $6(2.26)$ & $6(2.01)$ & $3(1.46)$ & $13(1.39)$ & 36 & 1.78 \\
\hline Total & 322 (15.88) & $266(13.12)$ & 299 (14.75) & 205 (10.11) & 935 (46.12) & 2027 & \\
\hline
\end{tabular}

*Recorded to be Plasmodium positive in the current study. 


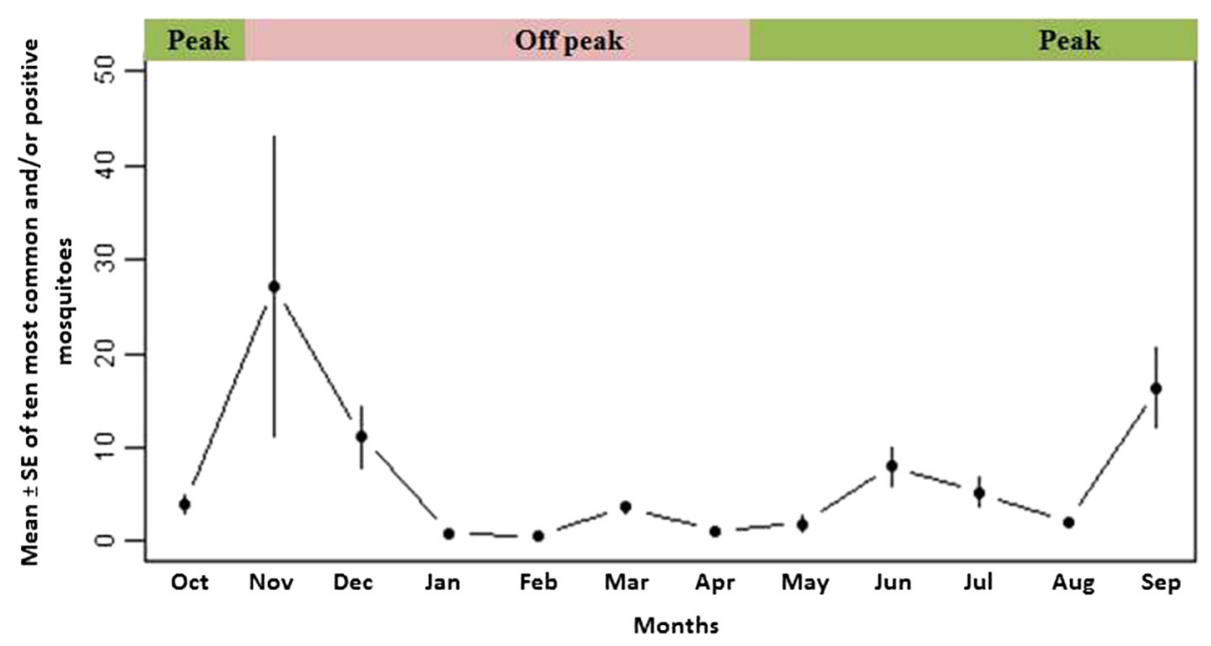

Figure 2 Temporal distribution of mean \pm SE of ten most common and/or positive Anopheles species Matiranga sub-district, Khagrachari.

and two An. karwari. A single An. kochi was observed to be infected with $\mathrm{Pv}-210$ while three additional mosquitoes (0.15\%) identified as $A n$. (A.) umbrosus Theobald, An. nivipes and An. kochi were positive for Pv-247. Additionally, one An. barbirostris s.l. was recorded positive for both Pf and Pv-247.

The abundance of mosquito species varied among villages. Shannon's species diversity was highest in Khedachhara $\left(\mathrm{H}^{\prime}=2.25\right)$ and evenness was highest in Natunpara $(E=0.83)$ (Table 4). Overall mosquito abundance varied significantly $(\mathrm{p}<0.05)$ among the villages while it was significantly greater in Azoddha $(\mathrm{p}<0.05)$ (Additional file 1: Tables S1 and S2). Azoddha was also observed to be significantly $(\mathrm{p}<0.05)$ different from other villages when the distances of villages from the international border was considered (Additional file 1: Tables S3, S4 and S5). In addition, An. kochi, An. vagus, An. barbirostris s.l., An. nivipes, An. peditaeniatus, An. jeyporiensis, An. (A.) nigerrimus Giles and An. varuna were significantly $(\mathrm{p}<0.05)$ more abundant in Azoddha (Additional file 1: Tables S6, S7, S8, S9, S10, S11, S12 and S13). Among the eight houses detected harbouring CSP positive mosquitoes, six were located in this village (Table 3).

\section{Discussion}

Twenty Anopheles species were recorded in Matiranga in this study, which is $25 \%$ higher than the number of species $(n=15)$ previously reported from this area during the peak transmission season [8]. Three recent studies in an ecologically and topologically similar region of Bandarban reported 20, 21 and 22 Anopheles species, respectively $[7,28,12]$. Another entomological study in the Tripura state of neighbouring India identified 13 Anopheles species during peak transmission in June [9]. The full-year trapping effort may be the reason for the higher species diversity observed in this study. Anopheles jeyporiensis was observed to be the dominant mosquito

Table 2 Shannon's diversity and evenness indices of collected Anopheles mosquitoes from October 2010 to September 2011

\begin{tabular}{|c|c|c|c|c|}
\hline Months & Species richness & Number of mosquitoes & Shannon's diversity index $\left(\mathrm{H}^{\prime}\right)$ & Evenness (E) \\
\hline October & 13 & 97 & 2.12 & 0.82 \\
\hline November & 17 & 679 & 1.94 & 0.68 \\
\hline December & 15 & 277 & 1.95 & 0.72 \\
\hline January & 5 & 22 & 1.34 & 0.83 \\
\hline February & 4 & 15 & 1.07 & 0.77 \\
\hline March & 13 & 90 & 1.92 & 0.74 \\
\hline April & 8 & 29 & 1.52 & 0.73 \\
\hline May & 7 & 47 & 1.76 & 0.91 \\
\hline June & 10 & 183 & 1.79 & 0.74 \\
\hline July & 11 & 131 & 1.78 & 0.74 \\
\hline August & 8 & 52 & 1.61 & 0.77 \\
\hline September & 13 & 405 & 2.03 & 0.79 \\
\hline
\end{tabular}




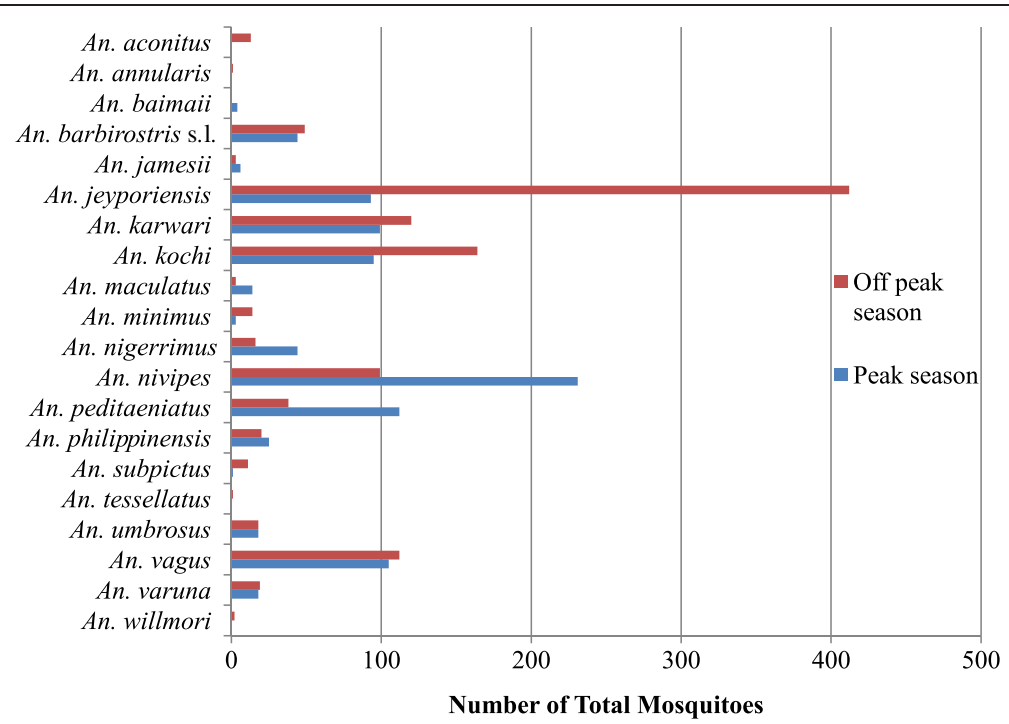

Figure 3 Relative abundance of Anopheles mosquitoes according to malaria transmission season.

species in the current study. As in a previous study in an ecologically similar area of Bandarban [7], An. jeyporiensis $(24.91 \%)$ was observed to be the dominant mosquito species overall. However, An. nivipes, the second most abundant species overall (16.18\%) was the most abundant species during the peak transmission season, echoing the findings of Alam et al. [7]. Overall, dominancy was observed from An. vagus (51.7\%) followed by $A n$. philippinensis (22.92\%) in another similar study in Bandarban [12].

Anopheles philippinensis is considered to be the most important malaria vector in the flood plains of Bangladesh [6]. Recent entomological investigations have reported the abundance of this species in CHT $[28,12]$, as well as in the Matiranga border adjacent to the north-eastern Indian state of Tripura [9]. However, the precise limits of the distribution of this species cannot be defined due to its common misidentification with An. nivipes. Alam et al. [7] confirmed all specimens morphologically identified as $A n$. philippinensis in Bandarban, to be An. nivipes by molecular assay. Nagpal and Sharma [29] also stated that previously identified $A n$. philippinensis from north eastern India were in fact $A n$. nivipes. In the present study, coexistence of $A n$. nivipes and An. philippinensis was confirmed in Matiranga. One specimen of morphologically identified An. philippinensis that was reported positive for $\mathrm{Pv}-210$ in the previous study in Matiranga [8], may have actually been $A n$. nivipes. The coexistence of two such closely related species in a malaria hyperendemic area indicates the importance of detailed entomological investigations. As previously reported from the CHT [7], all specimens of $A n$. minimus complex collected in the current study were confirmed as An. minimus by PCR.

Recent studies have implicated An. jeyporiensis, An. nivipes and $A n$. vagus as potential vectors of malaria

Table 3 Summary information of CSP ELISA positive Anopheles species and the distance of positive sites from Bangladesh-India international border

\begin{tabular}{|c|c|c|c|c|}
\hline Mosquito ID & Village & Species name & CSP ELISA & Distance (Kilometres) \\
\hline KS 0221 & Belchhari & An. karwari & Pf & 1.91 \\
\hline KS 0357 & Azoddha & An. vagus & Pf & 1.27 \\
\hline KS 0515 & Azoddha & An. kochi & Pv 210 & 1.23 \\
\hline KS 1004 & Aambagan & An. barbirostris s.l. & Pf & 3.12 \\
\hline KS 1051 & Khedachhara & An. karwari & Pf & 4.54 \\
\hline KS 1147 & Azoddha & An. barbirostris s.l. & Mixed $^{* *}$ & 1.2 \\
\hline KS 1154 & Azoddha & An. umbrosus & Pv 247 & 1.26 \\
\hline KS 1330 & Azoddha* & An. nivipes & Pv 247 & 0.71 \\
\hline KS 1347 & Azoddha* & An. kochi & Pv 247 & 0.71 \\
\hline
\end{tabular}


Table 4 Shannon's diversity and evenness of Anopheles species in five study villages of Matiranga sub-district, Khagrachari

\begin{tabular}{lllll}
\hline Village & Number of mosquitoes collected & Distance from international border* & Shannon's diversity index (H') & Evenness (E) \\
\hline Natunpara & 322 & $7.8 \mathrm{~km}$ & 2.18 & 0.83 \\
Aambagan & 266 & $3.6 \mathrm{~km}$ & 2.11 & 0.79 \\
Azoddha & 935 & $1 \mathrm{~km}$ & 2.14 & 0.71 \\
Belchhari & 205 & $2.2 \mathrm{~km}$ & 2.00 & 0.80 \\
Khedachhara & 299 & $4.7 \mathrm{~km}$ & 2.25 & 0.81 \\
\hline
\end{tabular}

*Distance from the international border to the middle point of the village.

parasites in the CHT [7,12]. These three species were similarly abundant in this surveillance study and one specimen each of $A n$. vagus and $A n$. nivipes were recorded positive for Pf and $\mathrm{Pv}-247$, respectively, confirming their role in malaria transmission in Matiranga. Similar to Bangladesh, these unimportant mosquitoes have also been recorded to be positive for Plasmodium in areas of the international borders of Bangladesh. Based on morphological identification, $A n$. jeyporiensis was recorded to be positive in a study conducted in Bangladesh-Myanmar border [30]. Molecular evidence had revealed the involvement of An. nivipes and An. vagus for malaria transmission in north-eastern India, which is adjacent to Matiranga, Khagrachari [31,32].

Plasmodium infection in An. karwari, An. kochi and $A n$. umbrosus observed in the present investigation corroborates previous studies $[7,8,12]$. Anopheles kochi has been implicated as a malaria vector of Assam in northeast India [32], and this species and An. karwari can be considered as vectors in Bangladesh [7,12]. Anopheles barbirostris s.l. was reported to be CSP positive in Sri Lanka for Pf [33], as well as in a previous investigation in Matiranga and Bandarban $[8,12]$. In the current study, two specimens of $A n$. barbirostris s.l. were CSP positive where one specimen was positive for Pf and a mixed infection of Pf and Pv-247 was recorded in the other. Such a mixed infection has not been reported before in $A n$. barbirostris s.l. from Bangladesh and is rarely reported in southeast Asia [34]. More studies need to be conducted on these species in order to define their precise role in malaria transmission.

Forests of the CHT have been converted to unused shrub lands, agricultural lands and human settlements over the last 30 years [11,35]. Deforestation can significantly alter the mosquito community by magnifying vector density and abundance in the deforested areas [36,37]. It is evident from the current study that, principal vector mosquitoes, such as $A n$. baimaii and $A n$. minimus were noted to be very low in abundance compared to more recently implicated vector species.

Increasing population growth and human encroachment into previously under-developed international border areas has resulted in a higher contact rate between human and vector mosquitoes, increasing malaria parasite transmission [38]. In the current study significantly higher mosquito abundance and more ELISA positive mosquitoes were recorded in Azoddha, which is situated within two kilometres of the Bangladeshi-Indian border. During field surveys this village was observed to be surrounded by rice fields, which made this village different than other villages. Since the flight range of most Anopheles species is believed to be approximately one kilometre $[26,18]$, malaria transmission dynamics in trans-border areas might be influenced by such local spatial variation of potential vector mosquitoes observed in this study.

\section{Conclusion}

Similar to other endemic areas of CHT, Matiranga has diverse Anopheles fauna. However, low densities of recognized vectors and comparatively greater abundance of previously underappreciated Anopheles species may play a significantly heightened role in malaria transmission in areas adjacent to the Bangladesh-India border.

\section{Additional file}

Additional file 1: Tables of the results from negative binomial regression.

Competing interests

The authors declare that they have no competing interests.

Authors' contributions

MSA, WAK, RH and DEN conceived and designed the study. HMA, RE and SC carried out the sample collection. HMA and SC worked on taxonomic identification. ANM, RE and HMA performed laboratory assays. HMA, RE, MAHK and JSL analyzed the data. HMA, MSA, RE and JSL drafted the manuscript. All authors read the final manuscript and approved.

\section{Acknowledgments}

This investigation received partial financial assistance from WHO/SEARO in collaboration with UNICEF/UNDP/World Bank/WHO Special Programme for Research and Training in Tropical Diseases (TDR). icddr,b also gratefully acknowledges the following donors which provide unrestricted support: Australian Agency for International Development (AusAID), Government of the People's Republic of Bangladesh, Canadian International Development Agency (CIDA), Swedish International Development Cooperation Agency (Sida) and the Department for International Development. UK (DFID). The authors are grateful to NMCP for their permission to conduct the study in 
their facilities. The authors are also grateful to Dr. Wirtz's laboratory at the CDC for generously providing CSP reagents.

The authors are also indebted to the people of Matiranga who allowed the installation of light traps inside their sleeping rooms and the doctors and staff of Matiranga UHC for their extended support. The authors also appreciate the valuable contributions of Dr. Abdul Jabber Howlader, Md. Sharif Hossain, Shariar Mustafa, Milka Patracia Podder, Khaja Md. Mohiuddin, Dr. Khairul Bashar and B. M. Rabiul Islam Ripon in the study.

\section{Author details}

'International Centre for Diarrhoeal Disease Research Bangladesh (icddr,b), 68 Shaheed Tajuddin Ahmed Sarani, Mohakhali, Dhaka 1212, Bangladesh. ${ }^{2}$ Department of Biochemistry, Virginia Tech, Blacksburg, VA 24061, USA. ${ }^{3}$ Department of Microbiology and Infectious Disease, Cumming School of Medicine, University of Calgary, Alberta T2N1N4, Canada. ${ }^{4}$ Liverpool School of Tropical Medicine, Pembroke Place, Liverpool L3 5QA, UK. Johns Hopkins Malaria Research Institute, Department of Molecular Microbiology and Immunology, Johns Hopkins University Bloomberg School of Public Health, Baltimore, MD 21205, USA.

\section{Received: 8 January 2015 Accepted: 17 March 2015}

\section{Published online: 01 April 2015}

\section{References}

1. WHO. World Malaria Report: 2014. Geneva: World Health Organization; 2014.

2. MOHFW. Health Bulletin 2014. Dhaka: Directorate General of Health Services 2014; 2014.

3. Ahmed S, Galagan S, Scobie H, Khyang J, Prue CS, Khan WA, et al. Malaria hotspots drive hypoendemic transmission in the Chittagong Hill Districts of Bangladesh. PLoS One. 2013;8(8):e69713.

4. Haque U, Overgaard HJ, Clements AC, Norris DE, Islam N, Karim J, et al. Malaria burden and control in Bangladesh and prospects for elimination: an epidemiological and economic assessment. Lancet Glob Health. 2014;2(2):e98-105.

5. Haque U, Ahmed SM, Hossain S, Huda M, Hossain A, Alam MS, et al. Malaria prevalence in endemic districts of Bangladesh. PLoS One. 2009;4(8):e6737.

6. Elias M, Dewan ZAR, Ahmed R. Vectors of malaria in Bangladesh. J Prev Social Med. 1982;1:20-8.

7. Alam MS, Chakma S, Khan WA, Glass GE, Mohon AN, Elahi R, et al. Diversity of anopheline species and their Plasmodium infection status in rural Bandarban, Bangladesh. Parasit Vectors. 2012;5:150.

8. Alam MS, Khan MG, Chaudhury N, Deloer S, Nazib F, Bangali AM, et al. Prevalence of anopheline species and their Plasmodium infection status in epidemic-prone border areas of Bangladesh. Malar J. 2010;9:15.

9. Prakash A, Bhattacharyya DR, Mohapatra PK, Mahanta J. Investigation on malaria vectors and mosquito fauna in south Tripura district, Tripura state. Indian J Malariol. 1998;35(3):151-9.

10. Dhiman S, Goswami D, Rabha B, Gopalakrishnan R, Baruah I, Singh L. Malaria epidemiology along Indo-Bangladesh border in Tripura State, India. Southeast Asian J Trop Med Public Health. 2010;41(6):1279-89.

11. Anonymous. Land Cover Dynamics in Greater Chittagong. http://apps. geoportal.icimod.org/BDLandcover/\#. Accessed 12 Nov 2014.

12. Bashar K, Tuno N. Seasonal abundance of Anopheles mosquitoes and their association with meteorological factors and malaria incidence in Bangladesh. Parasit Vectors. 2014;7:442.

13. Thompson R, Begtrup K, Cuamba N, Dgedge M, Mendis C, Gamage-Mendis A, et al. The Matola malaria project: a temporal and spatial study of malaria transmission and disease in a suburban area of Maputo, Mozambique. Am J Trop Med Hyg. 1997;57(5):550-9.

14. Besansky NJ. Genome analysis of vectorial capacity in major Anopheles vectors of malaria parasites. White paper submitted to the Eurkaryotic Pathogens and Disease Vectors Target Selection Group on behalf of the Anopheles Genomes Cluster Committee. 2008.

15. TDR. Report of the Scientific Working Group on Vector and Human Health. 2002.

16. BBS. Bangladesh Population Census-2011. Dhaka: Bangladesh Bureau of Statistics, Ministry of Planning: Bangladesh; 2013.

17. Peyton EL, Scanlon JE, Malikul V, Imvitaya S. Illustrated Key to the Female Anopheles Mosquitoes of Thailand. Bangkok: U.S. Army Medical Component, Department of Medical Entomology, South East Asia Treaty Organization, APO San Francisco, 96346, Entomology DoM; 1966.
18. Nagpal BN, Sharma VP. Indian Anophelines. 66 Janpath, New Delhi 110001 : Mohan Primlani for Oxford \& IBH Publishing Co. Pvt. Ltd; 1995.

19. Lardeux F, Tejerina R, Aliaga C, Ursic-Bedoya R, Lowenberger C, Chavez T. Optimization of a semi-nested multiplex PCR to identify Plasmodium parasites in wild-caught Anopheles in Bolivia, and its application to field epidemiological studies. Trans R Soc Trop Med Hyg. 2008;102(5):485-92.

20. Huong NT, Sonthayanon P, Ketterman AJ, Panyim S. A rapid polymerase chain reaction based method for identification of the Anopheles dirus sibling species. Southeast Asian J Trop Med Public Health. 2001;32(3):615-20.

21. Phuc HK, Ball AJ, Son L, Hanh NV, Tu ND, Lien NG, et al. Multiplex PCR assay for malaria vector Anopheles minimus and four related species in the Myzomyia Series from Southeast Asia. Med Vet Entomol. 2003;17(4):423-8.

22. Walton C, Somboon P, Harbach RE, Zhang S, Weerasinghe I, O'Loughlin SM, et al. Molecular identification of mosquito species in the Anopheles annularis group in southern Asia. Med Vet Entomol. 2007;21(1):30-5.

23. Walton C, Somboon P, O'Loughlin SM, Zhang S, Harbach RE, Linton YM, et al. Genetic diversity and molecular identification of mosquito species in the Anopheles maculatus group using the ITS2 region of rDNA. Infect Genet Evol. 2007;7(1):93-102

24. Wirtz RA, Zavala F, Charoenvit Y, Campbell GH, Burkot TR, Schneider I, et al. Comparative testing of monoclonal antibodies against Plasmodium falciparum sporozoites for ELISA development. Bull World Health Organ. 1987;65(1):39-45.

25. Shannon CE. A mathematical theory of communications. Bell Syst Techn J. 1948;27:379-423.

26. Ejercito A, Urbino CM. Flight range of gravid and newly emerged Anopheles. Bull World Health Organ. 1951;3(4):663.

27. Team RDC. R: A Language and Environment for Statistical Computing. The R Foundation for Statistical Computing. Vienna, Austria: R Foundation for Statistical Computing; 2011. p. R is a free software environment for statistical computing and graphics. It can be used to generate species distribution models using as a base data such as those made available through GBIF.

28. Bashar K, Tuno N, Ahmed TU, Howlader AJ. Blood-feeding patterns of Anopheles mosquitoes in a malaria-endemic area of Bangladesh. Parasit Vectors. 2012;5:39.

29. Nagpal BN, Sharma VP. Survey of mosquito fauna of northeastern region of India. Indian J Malariol. 1987;24(2):143-9.

30. Macan TT. Malaria survey of the Arakan region of Bengal and Burma. Parasitology. 1950;40(3 \& 4):290-7.

31. Bhattacharyya DR, Prakash A, Sarma NP, Mohapatra PK, Singh S, Sarma DK, et al. Molecular evidence for the involvement of Anopheles nivipes (Diptera: Culicidae) in the transmission of Plasmodium falciparum in north-eastern India. Ann Trop Med Parasitol. 2010;104(4):331-6.

32. Prakash A, Bhattacharyya DR, Mohapatra PK, Mahanta J. Role of the prevalent Anopheles species in the transmission of Plasmodium falciparum and P. vivax in Assam state, north-eastern India. Ann Trop Med Parasitol. 2004;98(6):559-68.

33. Amerasinghe PH, Amerasinghe FP, Konradsen F, Fonseka KT, Wirtz RA. Malaria vectors in a traditional dry zone village in Sri Lanka. Am J Trop Med Hyg. 1999;60(3):421-9.

34. Imwong M, Nakeesathit S, Day NP, White NJ. A review of mixed malaria species infections in anopheline mosquitoes. Malar J. 2011;10:253.

35. Uddin K, Gurung DR. Land cover change in Bangladesh-a knowledge based classification approach. 10th International Center for Integrated Mountain Development, Kathmandu (ICIMOD); 2010; Khumaltar, Lalitpur, Nepal.

36. Yasuoka J, Levins R. Impact of deforestation and agricultural development on anopheline ecology and malaria epidemiology. Am J Trop Med Hyg. 2007;76(3):450-60.

37. Pascual M, Ahumada JA, Chaves LF, Rodo X, Bouma M. Malaria resurgence in the East African highlands: temperature trends revisited. Proc Natl Acad Sci U S A. 2006;103(15):5829-34.

38. Bhumiratana A, Intarapuk A, Sorosjinda-Nunthawarasilp P, Maneekan P, Koyadun S. Border malaria associated with multidrug resistance on Thailand-Myanmar and Thailand-Cambodia borders: transmission dynamic, vulnerability, and surveillance. Biomed Res Int. 2013;2013:363417. 